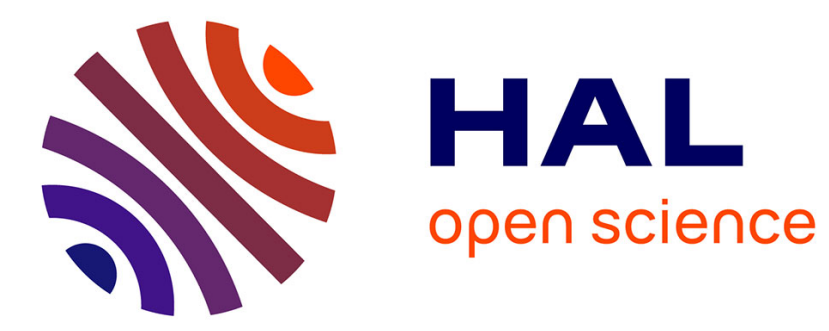

\title{
Generic validity of the multifractal formalism
}

Aurélia Fraysse

\section{To cite this version:}

Aurélia Fraysse. Generic validity of the multifractal formalism. SIAM Journal on Mathematical Analysis, 2007, 39 (2), pp.593-607. hal-00689978

\section{HAL Id: hal-00689978 https://hal.science/hal-00689978}

Submitted on 20 Apr 2012

HAL is a multi-disciplinary open access archive for the deposit and dissemination of scientific research documents, whether they are published or not. The documents may come from teaching and research institutions in France or abroad, or from public or private research centers.
L'archive ouverte pluridisciplinaire HAL, est destinée au dépôt et à la diffusion de documents scientifiques de niveau recherche, publiés ou non, émanant des établissements d'enseignement et de recherche français ou étrangers, des laboratoires publics ou privés. 


\title{
Generic validity of the multifractal formalism
}

\author{
A. Fraysse*
}

September 13, 2006

\begin{abstract}
The multifractal formalism is a conjecture which gives the spectrum of singularities of a signal using numerically computable quantities. We prove its generic validity by showing that almost every function in a given function space is multifractal and satisfies the multifractal formalism.
\end{abstract}

\section{Introduction}

One motivation of multifractal analysis was the study of fully developed turbulent flows. Indeed, some experimental results obtained in wind-tunnels showed that the regularity of the velocity of a turbulent fluid changes wildly from point to point. This quantity is therefore hardly computable. Hence, rather than measure the exponent at some point one rather estimates the fractal dimension of sets where it takes a given value $H$.

The spectrum of singularities $d(H)$ is the function which gives the Hausdorff dimension of those sets. From its definition, it is also almost impossible to obtain numerically the spectrum of singularities.

In [10], two physicists U. Frisch and G. Parisi proposed an algorithm in order to derive the spectrum of singularities from quantities that are effectively computable on a signal. They proposed to use the $L^{p}$ modulus of continuity of the velocity, used in the theory

${ }^{*}$ Laboratoire d'analyse et de mathématiques appliquées, Université Paris XII, 61 Avenue du Général de Gaulle, 94010 Créteil Cedex, FRANCE. Email: fraysse@univ-paris12.fr 
of turbulent flows since Kolmogorov, [18]. This average quantity is called the scaling function, or scaling exponent and is denoted $\xi_{f}$. It is defined by $\int|f(x+l)-f(x)|^{p} d x \sim|l|^{\xi_{f}(p)}$, where $\sim$ means that $\int|f(x+l)-f(x)|^{p} d x$ is of the order of magnitude of $|l|^{\xi_{f}(p)}$ when $l$ tends to 0 (assuming that the limit exists). Numerical estimations and further results about the scaling function and its wavelet decomposition can be found in $[1,2]$.

Frisch and Parisi proposed that the spectrum of singularities of a function can be obtained as follows:

$$
d(H)=\inf _{p \in \mathbb{R}}\left(p H-\xi_{f}(p)+d\right),
$$

see [10] for the derivation of this formula.

First, we state the mathematical framework of multifractal analysis. The main notion we need to define is the Hölder exponent.

Definition 1. Let $\alpha \geq 0$; a function $f: \mathbb{R}^{d} \rightarrow \mathbb{R}$ is $C^{\alpha}\left(x_{0}\right)$ if for all $x \in \mathbb{R}^{d}$ such that $\left|x-x_{0}\right| \leq 1$ there exists a polynomial $P$ of degree less than $[\alpha]$ and a constant $C$ such that,

$$
\left|f(x)-P\left(x-x_{0}\right)\right| \leq C\left|x-x_{0}\right|^{\alpha} .
$$

The Hölder exponent of $f$ at $x_{0}$ is

$$
h_{f}\left(x_{0}\right)=\sup \left\{\alpha: f \in C^{\alpha}\left(x_{0}\right)\right\} .
$$

It is proved in [14] that for $p \geq 1$, the scaling function $\xi_{f}(p)$ is closely related with Sobolev or Besov smoothness. It is thus natural for us to replace the scaling function as follows.

$$
\text { If } p>0 \quad \eta_{f}(p)=\sup \left\{s: f \in B_{p}^{s / p, \infty}\right\} .
$$

So (1) applied to $\eta_{f}$ can at most give the increasing part of the spectrum.

Defining, as in [16], an auxiliary function $s(1 / p)=\eta(p) / p$, the Besov domain of a function $f$ is the set of $(q, t)$ such that $f \in B_{1 / q}^{t, 1 / q}$. The boundary of the Besov domain of $f$ is then given by the graph of $s$. And by Sobolev embeddings, the Besov domain of a function is a convex set. Thus, functions $\eta$ satisfying (3) are increasing and concave functions. Furthermore the auxiliary function $s$ is such that $0 \leq s^{\prime}(q) \leq d$. Those conditions lead us to the following definition. 
Definition 2. A function $\eta$ is admissible if $s(q)=q \eta(1 / q)$ is concave and satisfies $0 \leq s^{\prime}(q) \leq d$. It is strongly admissible if furthermore $s(0)>0$.

The following important result from [16] allows us to define a metric space using admissible functions.

Proposition 1. Any concave function s satisfying $0 \leq s^{\prime}(q) \leq d$ defines the Besov domain of a distribution $f$.

Thanks to Proposition 1, to each admissible function $\eta$, a metric space $V$ can be associated by taking

$$
V=\bigcap_{\varepsilon>0,0<p<\infty} B_{p, l o c}^{(\eta(p)-\varepsilon) / p, p} .
$$

To be as complete as possible, we also recall the definition of Legendre transform.

Definition 3. Let $f$ be a lower semi-continuous function defined in a normed vector space $E$. Then the Legendre transform of $f$ is

$$
f^{*}(x)=\sup _{y \in E}(f(y)-x y) .
$$

This function is convex and lower semi-continuous.

In the present paper, we propose to study the validity of (1) for $\eta_{f}(p)$. An equivalent form of this heuristic formula is satisfied by a large class of invariant measures, see $[4,6,20]$. In the context of signal analysis, this conjecture is often satisfied if we add particular assumptions on $f$, such as self-similarity. On the other hand, there exist counterexamples to the general validity of this formula. If it does not hold for every function, what is its range of validity? Our purpose here is to show that the validity of formula (1) is not an exceptional phenomenon but it is satisfied for a large class of functions, without any additional assumption. More precisely, we study the validity of this formula for "almost every" functions, i.e. in a measure-theoretic sense.

In a finite dimensional space, the notion of "almost every" means "for the Lebesgue measure". The particular role played by this measure is justified by the fact that this is the only one which is $\sigma$-finite and invariant under translation. In a metric infinite dimensional space no measure enjoys this properties. The following definition, see $[5,7,13]$ can thus replace the notion of vanishing Haar measure. 
Definition 4. Let $V$ be a complete metric vector space. A Borel set $B$ in $V$ is called Haar null if there exists a probability measure $\mu$ with compact support such that

$$
\mu(B+v)=0 \quad \forall v \in V .
$$

In this case the measure $\mu$ is said transverse to $B$.

A subset of $V$ is called Haar null if it is contained in a Borel Haar null set.

The complement of a Haar null set is called a prevalent set.

With a slight abuse of language we will say that a property is satisfied almost everywhere when it holds on a prevalent set.

Let us recall some properties of Haar null sets, see [7, 13].

Proposition 2. 1. If $S$ is Haar-null, then $\forall x \in V, x+S$ is Haarnull.

2. If $\operatorname{dim}(V)<\infty, S$ is Haar-null if and only if meas $(S)=0$ (where meas denotes the Lebesgue measure).

3. Prevalent sets are dense.

4. If $S$ is Haar null and $S^{\prime} \subset S$ then $S^{\prime}$ is Haar null.

5. The union of a countable collection of Haar null sets is Haar null.

6. If $\operatorname{dim}(V)=\infty$, compact subsets of $V$ are Haar-null.

Several kinds of measures can be used as transverse measures of a Borel set. Here, we will only use the following notion.

Definition 5. A finite dimensional space $P$ is called a probe for the set $T \subset V$ if the Lebesgue measure on $P$ is transverse to the complement of $T$.

Those measures are not compactly supported probability measures. However one immediately checks that Definition 5 is equivalent to the same one stated with the Lebesgue measure defined on the unit ball of $P$. Note that in this case, the support of the measure is included in the unit ball of a finite dimensional subspace. The compactness assumption is therefore fulfilled. 
The study of generic regularity for a "large" set of functions goes back to S. Banach [3], who gave differentiability properties of continuous functions, for quasi-all functions in the Baire's categories sense. Later B. Hunt [12] proved the same result in the measure-theoretic sense of prevalence.

In [16], S. Jaffard studied properties of generic functions, in the Baire's categories sense, in Sobolev spaces. He also proved that in the sense of Baire's categories quasi-all functions in $V$ satisfy:

$$
d(H)=\inf _{p \geq p_{c}}(p H-\eta(p)+d)
$$

where $p_{c}$ is the only critical point such that $\eta(p)=d$.

In this paper we will study the validity of the Frisch-Parisi conjecture for almost every function in the prevalence setting. The aim of this paper is to prove the following theorem.

Theorem 1. Let $\eta$ be a strongly admissible function and let $V$ be the space defined by

$$
V=\bigcap_{\varepsilon>0,0<p<\infty} B_{p, l o c}^{(\eta(p)-\varepsilon) / p, p}
$$

then, in the sense of prevalence, almost every function $f$ in $V$ satisfies the following two conditions:

1. For all $p>0$,

$$
\eta_{f}(p)=\eta(p)
$$

2. The spectrum of singularities is defined on the interval $\left[s(0), \frac{d}{p_{c}}\right]$ where it is given by:

$$
d_{f}(H)=\inf _{p \geq p_{c}}\left(p H-\eta_{f}(p)+d\right)
$$

where $p_{c}$ is the only critical point such that $\eta\left(p_{c}\right)=d$.

Remark. We have to impose that $\eta$ is strongly admissible else, according to [8], almost every function in $V$ is nowhere locally bounded.

In section 2 we will solve a simpler problem. We will prove that almost every function in a given intersection of a Sobolev or a Besov space and an Hölder space satisfies a slight modification of the Frisch and Parisi conjecture. We will first establish their spectrum of singularities. 
Theorem 2. If $\gamma>0$ and $s-\frac{d}{p}<0$ the spectrum of singularities of almost every function in $B_{p}^{s, q} \cap C^{\gamma}$ or in $L^{p, s} \cap C^{\gamma}$ is given by:

$$
d(H)= \begin{cases}\frac{d+(\gamma-s) p}{\gamma} H & \text { if } H \in\left[\gamma, \frac{d \gamma}{d+(\gamma-s) p}\right] \\ -\infty & \text { otherwise. }\end{cases}
$$

Remarks. 1. Using the Sobolev embeddings $B_{p}^{s, 1} \hookrightarrow L^{p, s} \hookrightarrow B_{p}^{s, \infty}$, the same result holds in Sobolev and in Besov spaces. As Besov spaces have a very simple wavelet expansion, we will only prove the result in those spaces. To obtain the Sobolev case, we only need to pick $q=\infty$ in the following.

2. In Theorem 2 we only state the spectrum of singularities of functions in the case $B_{p}^{s, q} \cap C^{\gamma}$ where $s-\frac{d}{p}<0$. Other cases are proved in [9]. To be complete, we recall the following result from [9].

Proposition 3. - If $s-d / p \leq 0$, then almost every function in $L^{p, s}$ or in $B_{p}^{s, q}$ is nowhere locally bounded, and therefore its spectrum of singularities is not defined.

- If $s-d / p>0$, then the Hölder exponent of almost every function $f$ of $L^{p, s}$, or of $B_{p}^{s, q}$ takes values in $[s-d / p, s]$ and

$$
\forall H \in[s-d / p, s], \quad d_{f}(H)=H p-s p+d ;
$$

furthermore, for almost every $x, h_{f}(x)=s$.

Our purpose here is to expand the result of [9] in two directions. On one hand, we will work with an intersection of Besov spaces. On the other hand, we will see in the last part another stronger generic result, in the topological sense mentioned above.

The main tool that we will use in the following is the wavelet expansion of functions. First, it yields a simple characterization of functional spaces and it offer a simple condition for pointwise regularity. Let us recall some properties of wavelet expansion.

There exist $2^{d}-1$ oscillating functions $\left(\psi^{(i)}\right)_{i \in\left\{1, \ldots, 2^{d}-1\right\}}$ in the Schwartz class such that the functions

$$
2^{d j} \psi^{(i)}\left(2^{j} x-k\right), \quad j \in \mathbb{Z}, k \in \mathbb{Z}^{d}
$$

form an orthonormal basis of $L^{2}\left(\mathbb{R}^{d}\right)$, see [19]. Wavelets are indexed by dyadic cubes $\lambda=\left[\frac{k}{2^{j}} ; \frac{k+1}{2^{j}}\left[{ }^{d}\right.\right.$. Thus, any function $f \in L^{2}$ can be written:

$$
f(x)=\sum c_{j, k}^{(i)} \psi^{(i)}\left(2^{j} x-k\right)
$$


where

$$
c_{j, k}^{(i)}=2^{d j} \int f(x) \psi^{(i)}\left(2^{j} x-k\right) d x .
$$

(Note that we use an $L^{\infty}$ normalization instead of an $L^{2}$ one, which simplifies the formulas). If $p>1$ and $s>0$, Sobolev space have thus the following characterization, see [19]:

$$
f \in L^{p, s} \Leftrightarrow\left(\sum_{\lambda \in \Lambda}\left|c_{\lambda}\right|^{2}\left(1+4^{j s}\right) \chi_{\lambda}(x)\right)^{1 / 2} \in L^{p}\left(\mathbb{R}^{d}\right),
$$

where $\chi_{\lambda}(x)$ denotes the characteristic function of the cube $\lambda$ and $\Lambda$ is the set of all dyadics cubes. Homogeneous Besov spaces, which will also be considered, are characterized (for $p, q>0$ and $s \in \mathbb{R}$ ) by

$$
f \in B_{p}^{s, q} \Longleftrightarrow \sum_{j}\left(\sum_{\lambda \in \Lambda_{j}}\left|c_{\lambda}\right|^{p} 2^{(s p-d) j}\right)^{q / p} \leq C
$$

where $\Lambda_{j}$ denotes the set of dyadics cubes at scale $j$, see [19]. Note that, if $p \in] 0,1[$, Besov spaces are not Banach spaces since they are not locally convex but nonetheless are separable complete metric vector spaces.

Pointwise regularity can also be expressed in terms of a condition on wavelet coefficients, see [14].

Proposition 4. Let $x$ be in $\mathbb{R}^{d}$. If $f$ is in $C^{\alpha}(x)$ then there exists $c>0$ such that for all $\lambda$ :

$$
\left|c_{\lambda}\right| \leq c 2^{-\alpha j}\left(1+\left|2^{j} x-k\right|\right)^{\alpha} .
$$

\section{Multifractal formalism in a given Besov space}

The Frisch-Parisi conjecture gives the spectrum of singularities as the Legendre transform of the scaling function. We will determine the validity of this formula for measure theoretic generic functions in a given Besov space, in two steps. First we will prove Theorem 2, which one gives the spectrum of singularities of almost every function. Afterwards, we will give the prevalent scaling function. This allows us to merge the spectrum obtained with formula (1) applied to the scaling function. 


\subsection{Proof of Theorem 2}

Proposition 3 states that if $s-\frac{d}{p}<0$, almost every function in $B_{p}^{s, q}$ is nowhere locally bounded and the spectrum of singularities is not defined for any $H$. To define this spectrum, we need to assume a minimum uniform regularity. That is why, in the following, we choose $s-\frac{d}{p}<0$ and $0<\gamma<s$ and we study almost every function in $B_{p}^{s, q} \cap C^{\gamma}$.

Theorem 2.1 from [17], yields an upper bound of the spectrum of singularities.

Lemma 1. Let $s-\frac{d}{p}<0$. For all functions $f \in B_{p}^{s, q} \cap C^{\gamma}$, the Hausdorff dimension of the set $\left\{x: f \notin C^{\alpha}(x)\right\}$ is bounded by $\frac{d+(\gamma-s) p}{\gamma} \alpha$.

We need also the following definition.

Definition 6. Let $\alpha \in\left[1, \frac{d}{d+(\gamma-s) p}\right]$. A point $x_{0}$ belongs to $J_{\alpha}$ if there exists an infinite sequence $(j, k) \in \mathbb{N} \times\left\{0, \ldots, 2^{j}-1\right\}^{d}, k=\left(k_{1}, \ldots, k_{d}\right)$ such that for each $i=1, \ldots, d k_{i}$ can be written $l_{i} 2^{j-L}$ and:

$$
\frac{1}{2^{j}}+\left|x_{0}-\frac{k}{2^{j}}\right|<\frac{1}{2^{\alpha L}}
$$

where $L:=\left[\frac{(d+(\gamma-s) p) j}{d}\right]$. We define the exponent of approximation of $x$ as $\alpha^{\prime}(x)=\sup \left\{\alpha: x \in J_{\alpha}\right\}$.

In [15], it is proved that the Hausdorff dimension of $J_{\alpha}$ is $\frac{d}{\alpha}$.

Let $\alpha \in\left[1, \frac{d}{d+(\gamma-s) p}\right], \varepsilon>0$ and $n \in \mathbb{N}$ such that $N=2^{d n}>\frac{d}{\varepsilon \alpha}+1$ be fixed. We denote $H(\alpha)=\frac{d \gamma}{\alpha(d+(\gamma-s) p)}$ and $\beta(\alpha)=H(\alpha)+\varepsilon$. Each dyadic cube of size $2^{-d j}$ can be split into $2^{d n}$ subcubes $i(\lambda)$ with side $2^{-(j+n)}$. We define the probe $P$ spanned by $N$ functions $g^{r}$ with the following wavelet coefficients $d_{\lambda}^{r}$ :

$$
d_{\lambda}^{r}= \begin{cases}j^{-2 / q} 2^{-\gamma j} & \text { if each } k_{i} \text { is a multiple of } 2^{j-L} \text { and } r=i(\lambda) \\ 0 & \text { elsewhere }\end{cases}
$$

where for each $j$ we denote $L=\left[\frac{(d+(\gamma-s) p) j}{d}\right]$.

One can check that these functions $g^{r}$ belong to $B_{p}^{s, q} \cap C^{\gamma}$, see [16]. 
Let $J_{\alpha}(i, l)=\frac{l}{2^{i}}+\left[-\frac{1}{2^{\alpha L}} \frac{1}{2^{\alpha L}}\right]^{d}$.

Let us first check that the set of points $S_{c}(\alpha)$ defined by $S_{c}(\alpha)=\left\{f=\sum c_{\lambda} \psi_{\lambda} \in B_{p}^{s, q} \cap C^{\gamma}: \exists x \in J_{\alpha} \forall j, k\left|c_{\lambda}\right| \leq c 2^{-\beta(\alpha) j}\left(1+\left|2^{j} x-k\right|\right)^{\beta(\alpha)}\right\}$.

is a Haar null Borel set. Indeed this set can be included in the lim sup on $i$ of the countable union over $l$ of sets:

$S_{c}(\alpha)^{i, l}=\left\{f=\sum c_{\lambda} \psi_{\lambda} \in B_{p}^{s, q} \cap C^{\gamma}: \exists x \in J_{\alpha}(i, l) \quad \forall j, k\left|c_{\lambda}\right| \leq c 2^{-\beta(\alpha) j}\left(1+\left|2^{j} x-k\right|\right)^{\beta(\alpha)}\right\}$.

which are closed sets.

We pick a sequence of functions $f_{n}$ in $S_{c}(\alpha)^{i, l}$ and such that $f_{n}$ converges to $f$ in $B_{p}^{s, q} \cap C^{\gamma}$. For each $n$, there exists $x_{n}$ in $J_{\alpha}(i, l)$ such that $f_{n}$ satisfies condition (12) at $x_{n}$. But $J_{\alpha}(i, l)$ is a compact set, so there exists $x \in J_{\alpha}(i, l)$ and a subsequence $\left(x_{n(i)}\right)_{i \in \mathbb{N}}$ such that $x_{n(i)}$ converges to $x$. As the mapping which gives wavelet coefficients of a function is continuous, $f$ satisfies also (12) at $x$.

Let $f \in B_{p}^{s, q} \cap C^{\gamma}$ be fixed. Consider the affine subset $M=\{\delta \in$ $\left.\mathbb{R}^{N} ; \quad f+\sum \delta^{i} g^{i} \in S_{c}(\alpha)\right\}$. Let $\delta_{1}$ and $\delta_{2}$ be in $M$. There exists $x_{1} \in J_{\alpha}$ and $x_{2} \in J_{\alpha}$ such that for $l=1,2$ :

$$
\left|c_{\lambda}+\sum \delta_{l}^{i} d_{\lambda}^{i}\right| \leq c 2^{-\beta(\alpha) j}\left(1+\left|2^{j} x-k\right|\right)^{\beta(\alpha)} \leq c 2^{-\alpha \beta(\alpha) L} .
$$

Furthermore $H(\alpha)>\gamma$ and, if $\lambda$ is such that each $k$ is a multiple of $2^{j-L}$.

$$
\left|d_{\lambda}^{i}\right|>\frac{1}{j^{2 / q}} 2^{-H(\alpha) L}
$$

So, taking (15) and (16) we obtain:

$$
\left\|\delta_{1}-\delta_{2}\right\|_{\mathbb{R}^{N}} \leq 2 c 2^{-\alpha \beta(\alpha) L} 2^{H(\alpha) L} j^{2 / q}=2 c j^{2 / q} 2^{-\alpha \varepsilon L} .
$$

When $j$ tends to infinity, the Lebesgue measure of $S_{c}(\alpha)$ tends to zero. Now, we take the countable union over $c$ and $\varepsilon_{n} \rightarrow 0$. As Haar null set are stable under inclusion, we obtain:

$$
\forall \alpha \in\left[1, \frac{d}{d+(\gamma-s) p}\right] \text { a.e. in } B_{p}^{s, q} \cap C^{\gamma} \forall x \in J_{\alpha} h_{f}(x) \leq H(\alpha) \text {. }
$$

Let $\left(\alpha_{n}\right)$ be a dense sequence in $\left[1, \frac{d}{d+(\gamma-s) p}\right]$. As a countable union of Haar null sets is still a Haar null set, for almost every function in $B_{p}^{s, q} \cap C^{\gamma}$,

$$
h_{f}(x) \leq H\left(\alpha_{n}\right) \quad \forall n \forall x \in J_{\alpha_{n}}
$$


Let $f$ be a function satisfying (17). Let $\alpha$ be fixed, there exists a nondecreasing subsequence $\left(\alpha_{\varphi_{n}}\right)$ which converges to $\alpha$ and the intersection of the subsets $J_{\alpha_{\varphi_{n}}}\left(:=\tilde{J}_{\alpha}\right)$ contains $J_{\alpha}$. Furthermore there exist a measure such that any set of dimension less than $d / \alpha$ is of measure zero. And the measure of $J_{\alpha}$ is positive. If $G_{H}=\left\{x: h_{f}(x) \leq\right.$ $H$ \}, with Lemma 1 we have that the Hausdorff dimension of $G_{H}$ is $\frac{d+(\gamma-s) p}{\gamma} H$. And the $\frac{d}{\alpha}$ Hausdorff measure of the set $\left\{x: h_{f}(x)<H\right\}$ equals zero. This way for almost every function in $B_{p}^{s, q} \cap C^{\gamma}$,

$$
d(H)=\frac{d+(\gamma-s) p}{\gamma} H \quad \text { for } H \in\left[\gamma, \frac{d \gamma}{d+(\gamma-s) p}\right]
$$

\subsection{The scaling function}

Let us now determine the scaling function of almost every function in a given Besov space. We will now show the following result.

Proposition 5. Let $s_{0}$ and $p_{0}$ be fixed such that $s_{0}-\frac{d}{p_{0}}>0$. Outside a Haar null set in $B_{p_{0}}^{s_{0}, \infty}$, we have:

$$
\eta_{f}(p)=\left\{\begin{array}{lc}
p s_{0} & p \leq p_{0} \\
d+p\left(s_{0}-\frac{d}{p_{0}}\right) & p \geq p_{0} .
\end{array}\right.
$$

Let $0<\gamma<s$ be fixed. If $s_{0}-\frac{d}{p_{0}}<0$, then outside a countable union of compact set in $B_{p_{0}}^{s_{0}, p_{0}} \cap C^{\gamma}$ :

$$
\eta_{f}(p)=\left\{\begin{array}{lc}
p s_{0} & p \leq p_{0} \\
\gamma p+p_{0}\left(s_{0}-\gamma\right) & p \geq p_{0} .
\end{array}\right.
$$

Proof: In each case, we can find in [21] the lower bound. Indeed, this bound is given by the Sobolev embedding.

To prove the upper bound, we will first consider the case $s_{0}-\frac{d}{p_{0}}>$ 0 . Let $\varepsilon>0$ be fixed and denote

$$
\tilde{s}(p)=\left\{\begin{array}{lc}
p s_{0}+\varepsilon & p \leq p_{0} \\
d+p\left(s_{0}-\frac{d}{p_{0}}\right)+\varepsilon & p \geq p_{0} .
\end{array} .\right.
$$

Let $0<p<\infty$ be fixed. We want to show that the set of functions belonging to $B_{p}^{\tilde{s}(p), \infty}$ for all $0<p<\infty$ is Haar null. This set is clearly 
closed and Borel. Let $j \geq 1$ and $k \in\left\{0, \ldots, 2^{j}-1\right\}^{d}$. We define $J \leq j$ and $K \in \mathbb{Z}^{d}$ such that

$$
\frac{K}{2^{J}}=\frac{k}{2^{j}}
$$

is an irreducible fraction. Let $a>\frac{3}{p_{0}}$. We define a probe spanned by the function $F$ with the following wavelet coefficients:

$$
d_{\lambda}=j^{-a} 2^{\left(\frac{d}{p_{0}}-s_{0}\right) j} 2^{-\frac{d}{p_{0}} J} .
$$

This function belongs to $B_{p_{0}}^{s_{0}, p_{0}}$.

Let $f$ be in $B_{p_{0}}^{s_{0}, p_{0}}$ and consider the affine subset

$$
M=\left\{\alpha \in \mathbb{R} ; f+\alpha F \in B_{p}^{\tilde{s}(p), \infty}\right\} .
$$

Suppose that there exist $\alpha_{1}$ and $\alpha_{2}$ in $M$. We have then three cases, following position of $p$.

- If $p=p_{0}$, then $\tilde{s}(p)=p_{0}+\varepsilon$ and

$$
\begin{aligned}
\left\|f+\alpha_{1} F-\left(f+\alpha_{2} F\right)\right\|_{B_{p}^{\tilde{s}(p), \infty}} & =\sup _{j} \sum_{k \in\left\{0, \ldots, 2^{j}-1\right\}^{d}}\left|\frac{\alpha_{1}-\alpha_{2}}{j^{a}} 2^{\left(\tilde{s}-\frac{d}{p_{0}}\right) j} 2^{\left(\frac{d}{p_{0}}-s_{0}\right) j} 2^{-\frac{d}{p_{0}} J}\right|^{p_{0}} \\
& =\sup _{j}\left|\frac{\alpha_{1}-\alpha_{2}}{j^{a}}\right| 2^{p_{0} \varepsilon j} \sum_{J=0}^{j} \sum_{K \in\left\{0, \ldots, 2^{J}-1\right\}^{d}} 2^{-d J} \\
& =\sup _{j} \frac{\left|\alpha_{1}-\alpha_{2}\right|}{j^{a}} 2^{p_{0} \varepsilon j} .
\end{aligned}
$$

But if $\alpha_{1}$ and $\alpha_{2}$ belong to $M$, this implies that

$$
f+\alpha_{1} F-\left(f+\alpha_{2} F\right) \text { belong to } B_{p}^{\tilde{s}(p), \infty} .
$$

This is possible only if $\alpha_{1}=\alpha_{2}$.

- If $p>p_{0}$, then $\tilde{s}(p)=d+p\left(s_{0}-\frac{d}{p_{0}}\right)+\varepsilon$. In this case,

$$
f+\alpha_{1} F-\left(f+\alpha_{2} F\right) \in B_{p}^{\tilde{s}(p), \infty}
$$

implies that there exist $c>0$ such that:

$\left\|f+\alpha_{1} F-\left(f+\alpha_{2} F\right)\right\|_{B_{p}^{\tilde{s}(p), \infty}} \leq\left\|f+\alpha_{1} F\right\|_{B_{p}^{\tilde{s}(p), \infty}}+\left\|f+\alpha_{2} F\right\|_{B_{p}^{\tilde{s}(p), \infty}} \leq c$.

We have then the following inequalities: 


$$
\begin{array}{r}
\forall j>0 \quad \sum_{k \in\left\{0, \ldots, 2^{j}-1\right\}^{d}}\left|\frac{\alpha_{1}-\alpha_{2}}{j^{a}} 2^{\left(\tilde{s}-\frac{d}{p}\right) j} 2^{\left(\frac{d}{p_{0}}-s_{0}\right) j} 2^{-\frac{d}{p_{0}} J}\right|^{p} \leq c \\
\forall j>0 \quad\left|\frac{\alpha_{1}-\alpha_{2}}{j^{a}}\right| 2^{p} 2^{\left(\tilde{s}-\frac{d}{p}\right) p j} 2^{\left(\frac{d}{p_{0}}-s_{0}\right) p j} \sum_{J=0}^{j} \sum_{K \in\left\{0, \ldots, 2^{J}-1\right\}^{d}} 2^{-\frac{d p}{p_{0}} J} \leq c
\end{array}
$$

By definition of $J$. And

$$
\begin{gathered}
\forall j>0\left|\frac{\alpha_{1}-\alpha_{2}}{j^{a}}\right|^{p} \sum_{J=0}^{j} 2^{\left(d-\frac{d p}{p_{0}}\right) J} \leq c 2^{\left(-\tilde{s}+\frac{d}{p}-\frac{d}{p_{0}}+s_{0}\right) p j} \\
\forall j>0\left|\frac{\alpha_{1}-\alpha_{2}}{j^{a}}\right| \leq c 2^{\left(-\tilde{s}+\frac{d}{p}-\frac{d}{p_{0}}+s_{0}\right) j}\left|\frac{1}{1-2^{j\left(d-d \frac{p}{p_{0}}\right)}}\right|^{\frac{1}{p}}
\end{gathered}
$$

As $p>p_{0}, 1-2^{j\left(d-d \frac{p}{p_{0}}\right)}$ is equivalent to 1 for large $j$ and (2.2) implies

$$
\left|\alpha_{1}-\alpha_{2}\right| \leq c j^{a} 2^{-\varepsilon j}
$$

which tends to zero when $j$ tends to infinity.

- If $p<p_{0}$, then $\tilde{s}(p)=s_{0}+\varepsilon$ and $1-2^{j\left(d-d \frac{p}{p_{0}}\right)}$ is equivalent to $2^{j\left(d-d \frac{p}{p_{0}}\right)}$ when $j$ tends to infinity. Thus in (2.2), we obtain again

$$
\left|\alpha_{1}-\alpha_{2}\right| \leq c j^{a} 2^{-\varepsilon j}
$$

In each case we have obtained that $M$ is of Lebesgue measure zero. Taking countable union over $\varepsilon \rightarrow 0$, and over $p$, we obtain the desired scaling exponent.

The second case, for $s_{0}-\frac{d}{p_{0}}<0$ can be treated the same way for $p \leq p_{0}$. The case $p>p_{0}$ is obtained taking the function which coefficients are given by (14) instead of $F$.

From Theorem 2 and Proposition 5, we obtain the following Legendre transform of the scaling function of almost every function in a given Besov space.

Proposition 6. Let $s_{0}>0$ and $0 \leq p_{0}<\infty$. 
- If $s_{0}-\frac{d}{p_{0}}>0$, then for almost every function in $B_{p}^{s, q}$ :

$$
\forall H \in\left[s_{0}-\frac{d}{p_{0}}, s_{0}\right] \quad \inf _{p>0}(d-\eta(p)+H p)=d-p_{0} s_{0}+H p_{0} .
$$

- If $s_{0}-\frac{d}{p_{0}}<0$, then for almost every function in $B_{p}^{s, q} \cap C^{\gamma}$ we have:

$$
\forall H \in\left[\gamma, s_{0}\right] \quad \inf _{p>0}(d-\eta(p)+H p)=d-p_{0} s_{0}+H p_{0} .
$$

This proposition shows that for $s_{0}-\frac{d}{p_{0}}>0$, the increasing part of the spectrum given by Frisch-Parisi conjecture is valid for almost every function. But for $s_{0}-\frac{d}{p_{0}}<0$, this Legendre transform does not correspond to the spectrum of singularities given by Theorem 2 .

\section{The Frisch-Parisi conjecture}

We will now prove Theorem 1. Instead of $B_{p_{0}}^{s_{0}, q_{0}}$ we will now work with:

$$
V=\bigcap_{\varepsilon>0,0<p<\infty} B_{p, l o c}^{(\eta(p)-\varepsilon) / p, p}
$$

This set $V$ can also be written as a countable intersection over $B_{p_{n}, l o c}^{\left(\eta\left(p_{n}\right)-\varepsilon_{n}\right) / p_{n}, p_{n}}$

Note that $V$ is a topological vector space. For $p<1$ Besov spaces are only quasi-Banach spaces, as the triangle inequality is only satisfied up to a constant, $V$ is not a Banach space but a complete metric space. Indeed, if $p \geq 1$ we take for distance between two functions $f$ and $g$ in $B_{p}^{s, q}$ :

$$
d(f, g)=\sum_{j \geq 0}\left(\sum_{k \in\left\{0, \ldots, 2^{j}-1\right\}^{d}}\left|\left(c_{j, k}-d_{j, k}\right) 2^{\left(s-\frac{d}{p}\right) j}\right|^{p}\right)^{\frac{q}{p}}
$$

where $c_{j, k}$ are the wavelet coefficients of $f$ and $d_{j, k}$ are those of $g$. If $p<1$ Besov spaces are not Banach spaces, but complete metric space with the following distance:

$$
d(f, g)=\left(\sum_{j \geq 0}\left(\sum_{k \in\left\{0, \ldots, 2^{j}-1\right\}^{d}}\left|\left(c_{j, k}-d_{j, k}\right) 2^{\left(s-\frac{d}{p}\right) j}\right|^{p}\right)^{\frac{q}{p}}\right)^{\frac{\min (p, q)}{q}} .
$$


Thus, we obtain a distance in $V$ taking:

$$
\forall f, g \in V \quad d(f, g)=\sum_{n} 2^{-n} \frac{d_{n}(f, g)}{1+d_{n}(f, g)}
$$

where $d_{n}$ denotes the distance in $B_{p_{n}, l o c}^{\left(\eta\left(p_{n}\right)-\varepsilon_{n}\right) / p_{n}, p_{n}}$. With this distance $V$ is clearly a complete space. Note that the measure used is the Lebesgue measure in the unit ball of a probe, so this is a probability measure with compact support.

In the following subsection we prove that the spectrum of singularities of almost every function in $V$ satisfies:

$$
d(H)=\inf _{p \geq p_{c}}(p H-\eta(p)+d) .
$$

\subsection{Proof of Theorem 1}

Let us now study the spectrum of singularities on a prevalent set of functions in $V$.

Proposition 7. For almost every function $f \in V$, the spectrum of singularities satisfies:

$$
\forall H \in\left[s(0), \frac{d}{p_{c}}\right] \quad d(H)=\inf _{p \geq p_{c}}(H p-\eta(p)+d) .
$$

Proof:

We will first construct the probe. Denote:

$$
a(j, k)=\inf _{p}\left(\frac{d(j-J)-\eta(p) j}{p}\right)
$$

and define $g$ via its wavelet coefficients:

$$
d_{\lambda}=\frac{1}{j^{a}} 2^{a(j, k)}
$$

where we denote $a=a_{j}=\log j$ and $J \leq j$ is such that there exists $K \in \mathbb{Z}^{d}$ and $\frac{k}{2^{j}}=\frac{K}{2^{j}}$ is an irreducible form.

First, we check that $g$ belongs to $V$. Let $p>0$ be fixed. Thus we have to show that $g \in B_{p}^{\eta(p) / p, \infty}$. Let $s=\frac{\eta(p)}{p}$. Since $a(j, k) \leq \frac{d(j-J)}{p}-s j$, $p a(j, k)+(\eta(p)-p) j=-J d$ and $g \in B_{p}^{\eta(p) / p, \infty}$. For further details upon this function $g$, we refer to [16]. 
Definition 7. Let $\alpha$ be fixed. We denote

$$
F_{\alpha}=\left\{x: \exists \text { a sequence }\left(\left(k_{n}, j_{n}\right)\right)_{n \in \mathbb{N}}\left|x-\frac{k_{n}}{2^{j_{n}}}\right| \leq \frac{1}{2^{\alpha j_{n}}}\right\} \text {. }
$$

The dyadic exponent of $x$ is defined by $\alpha\left(x_{0}\right)=\sup \left\{\alpha: x_{0}\right.$ is $\alpha$ approximable by dyadics $\}$

As it is stated in [16], the Hausdorff dimension of the set $F_{\alpha}$ is at least $\frac{d}{\alpha}$.

First, let $\alpha \in(1, \infty)$ be fixed and let $F_{\alpha}$ be the set given by Definition 7 . Let $\varepsilon>0$ be fixed, and let

$$
H(\alpha)=\frac{1}{\alpha} \sup _{\omega \geq \alpha}\left(\omega \sup _{q>0}\left(s(q)-d\left(1-\frac{1}{\omega}\right) q\right)\right)
$$

and $\gamma=\gamma(\alpha)=H(\alpha)+\varepsilon$.

Let $n \in \mathbb{N}$ be such that $N=2^{d n}>\frac{d}{\varepsilon}+1$ be fixed. The probe $P$ is spanned by $N$ functions $g_{i}$ which are deduced from $g$ by taking its wavelets coefficients only over some sub-cubes $i(\lambda)$ with size $2^{-d(j+n)}$. The aim of this part is to prove that the set of functions $f$ such that there exist a point in $F_{\alpha}$ where $f$ is $C^{\gamma}$ is a Haar null set. This set is included in the countable union of:

$S_{c}(\alpha)=\left\{f=\sum c_{\lambda} \psi_{\lambda}: \exists x \in F_{\alpha} \forall j, k\left|c_{\lambda}\right| \leq c 2^{-\gamma(\alpha) j}\left(1+\left|2^{j} x-k\right|\right)^{\gamma(\alpha)}\right\}$.

We can find a subsequence $(j, k)$ such that $J \leq \alpha j$ and:

$$
H(\alpha)=\frac{1}{\alpha} \sup _{\omega \geq \alpha}\left(\omega \sup _{q>0}\left(s(q)-d\left(1-\frac{1}{\omega}\right) q\right)\right) \geq-a(j, k) .
$$

If $x \in F_{\alpha}$ is fixed and $\lambda$ is such that $|x-\lambda| \leq A$ for $A>2 N$, the wavelet coefficients of $g_{i}$ satisfy:

$$
\left|d_{\lambda}^{i}\right| \geq \frac{c(A)}{j^{a}} 2^{-H(\alpha) j} .
$$

We will now prove that the set $S_{c}(\alpha)$ is a Borel Haar null set. First, this set is included in the countable union over $\lambda$ of:

$S_{c}(\alpha)^{j, k}=\left\{f=\sum c_{\lambda} \psi_{\lambda}: \exists x \in F_{\alpha}^{j, k} \forall j, k\left|c_{\lambda}\right| \leq c 2^{-\gamma(\alpha) j}\left(1+\left|2^{j} x-k\right|\right)^{\gamma(\alpha)}\right\}$.

Where $F_{\alpha}^{j, k}=\left\{x:\left|x-\frac{k}{2^{j}}\right| \leq \frac{1}{2^{\alpha j}}\right\}$. This set $S_{c}(\alpha)^{j, k}$ is a closed set and $S_{c}(\alpha)$ is a Borel set. Let $f$ be in $V$ and $\beta_{1}$ and $\beta_{2}$ be such that 
the functions $f+\sum \beta_{1}^{i} g^{i}$ and $f+\sum \beta_{2}^{i} g^{i}$ are in $S_{c}(\alpha)$. There exist two points $x_{1}$ and $x_{2}$ in $F_{\alpha}$ such that in the cone of influence above $x_{1}$ and $x_{2}$ :

$$
\left|c_{\lambda}+\sum \beta_{1}^{i} d_{\lambda}^{i}-\left(c_{\lambda}+\sum \beta_{2}^{i} d_{\lambda}^{i}\right)\right| \leq 2 c 2^{-\gamma(\alpha) j}
$$

Or,

$$
\left|c_{\lambda}+\sum \beta_{1}^{i} d_{\lambda}^{i}-\left(c_{\lambda}+\sum \beta_{2}^{i} d_{\lambda}^{i}\right)\right|=\left|\sum \beta_{1}^{i} d_{\lambda}^{i}-\beta_{2}^{i} d_{\lambda}^{i}\right|
$$

but, using (26),

$$
\left|\sum \beta_{1}^{i} d_{\lambda}^{i}-\beta_{2}^{i} d_{\lambda}^{i}\right| \geq\left|\sum \beta_{1}^{i}-\beta_{2}^{i}\right| \frac{c(A)}{j^{a}} 2^{-H(\alpha) j} .
$$

Thus,

$$
\left\|\beta_{1}-\beta_{2}\right\|_{\mathbb{R}^{N}} \leq \tilde{c} j^{a} 2^{-\varepsilon j} .
$$

So the Lebesgue measure in $\mathbb{R}^{N}$ of the set $\left\{\beta: f+\beta g \in S_{c}(\alpha)^{j, k}\right\}$ is bounded by $\left(\tilde{c} j^{a}\right)^{N} 2^{-N \varepsilon j}$.

The Lebesgue measure of the set of $\beta$ such that $f+\sum \beta^{i} g^{i}$ belongs to $S_{c}(\alpha)$ vanishes. Therefore $S_{c}(\alpha)$ is Haar null.

Taking a countable union over $c_{n}>0$ of sets $S_{c}(\alpha)$, the set of functions in $V$ with a pointwise Hölder exponent greater than $\gamma(\alpha)$ at a point of $F_{\alpha}$ is also Haar null. If $\varepsilon_{n} \rightarrow 0$, taking the union over $\varepsilon_{n}$ it follows that for all $\alpha \geq 1$ the set of functions in $V$ with an Hölder exponent greater than $H(\alpha)$ at some point of $F_{\alpha}$ is Haar null.

Let $\alpha_{n}$ be a dense sequence in $(1, \infty)$. By countable intersection:

$$
M=\left\{f \in V: \forall n \quad \forall x \in F_{\alpha_{n}} h_{f}(x) \leq H(\alpha)\right\}
$$

is prevalent. Let $f \in M$ and let $\alpha \geq 1$. There exist a subsequence $\alpha_{\phi(n)}$ which is nondecreasing and tends to $\alpha$. If we denote $\tilde{F}_{\alpha}$ the intersection of sets $F_{\alpha_{n}}$, it follows that $\tilde{F}_{\alpha}$ contains $F_{\alpha}$. Furthermore, the Hausdorff dimension of $\tilde{F}_{\alpha}$ is greater than $\frac{d}{\alpha}$ and for all $x \in \tilde{F}_{\alpha}$, $h_{f}(x) \leq H(\alpha)$. Finally we obtain

To conclude the second point of Theorem 1 , we rewrite $H(\alpha)$ in the following form

$$
H(\alpha)=\frac{1}{\alpha} \inf _{a \geq \alpha} G(a)
$$

where $G(a)=\sup _{q}(a(-q d+s(q))+q d)=a \sup _{q}\left(q d\left(-1+\frac{1}{a}\right)+s(q)\right)=$ $a s^{*}\left(d\left(1-\frac{1}{a}\right)\right)$. Here $s^{*}$ is the Legendre transform of $s$. By definition of the Legendre transform, this is a convex function. Furthermore it satisfies

$$
\left\{\begin{array}{ll}
s^{*}(h)=+\infty & \text { if } h<s^{\prime}(+\infty) \\
s^{*}(h)=s(0) & \text { if } h>s^{\prime}(0)
\end{array} .\right.
$$


And if $s^{*}$ is twice differentiable (we refer to [11] for a general case), $G$ is also twice differentiable and its derivative is

$$
G^{\prime}(a)=s^{*}\left(d\left(1-\frac{1}{a}\right)\right)+\frac{d}{a}\left(s^{*}\right)^{\prime}\left(d\left(1-\frac{1}{a}\right)\right)
$$

and:

$$
G^{\prime \prime}(a) \geq 0 .
$$

Thus $G$ is also convex and there exists $a_{0}$ such that $G\left(a_{0}\right)=\inf _{a \geq 0} G(a)$, $a_{0}$ being such that $G^{\prime}\left(a_{0}\right)=0$. We also deduce from (28) that:

$$
\left\{\begin{array}{ll}
G(a)=+\infty & \text { si } a<\frac{d}{d-s^{\prime}(+\infty)} \\
G(a)=a s(0) & \text { si } a>\frac{d}{d-s^{\prime}(0)}
\end{array} .\right.
$$

By definition of $s$ and with the hypothesis that $\eta$ is an admissible function we have $0 \leq s^{\prime}(q) \leq d$, for all $q>0$. It follows from (29) that $a_{0}$ belongs to the interval $\left(\frac{d}{d-s^{\prime}(+\infty)}, \frac{d}{d-s^{\prime}(0)}\right)$ which is included in $[0, \infty)$.

Another way to treat $G$ is to write $G(a)=\sup _{q}(\tilde{s}(q))$ where $\tilde{s}(q)=a(s(q)-q d)+q d$. And if $s$ is also twice differentiable, $\tilde{s}^{\prime}(q)=$ $-d a+a s^{\prime}(q)+d$ and $\tilde{s}^{\prime \prime}(q)=a s^{\prime \prime}(q)<0$. Thus $\tilde{s}$ is a concave function, and there exists an upper bound $q_{0}$ which satisfies $\tilde{s}^{\prime}\left(q_{0}\right)=$ $-d a+a s^{\prime}\left(q_{0}\right)+d=0$, and $s^{\prime}\left(q_{0}\right)=\frac{d a-d}{a}$. The value of $q_{0}$ also depends of $a$ so we write now $q_{0}=q(a)$.

We can finally write $G(a)=a(-q(a) d+s(q(a)))+q(a) d$. This function is twice differentiable and its derivative satisfies:

$$
G^{\prime}(a)=s(q(a))-d q(a) .
$$

If $a=a_{0}$ is the lower bound of $G$, we obtain $G^{\prime}\left(a_{0}\right)=s\left(q\left(a_{0}\right)\right)-$ $d q\left(a_{0}\right)=0 \Rightarrow s\left(q\left(a_{0}\right)\right)=d q\left(a_{0}\right)$, that is $q\left(a_{0}\right)=q_{c}=1 / p_{c}$. Furthermore, $G$ is decreasing for $a \leq a_{0}$ and increasing for $a \geq a_{0}$. The following cases are now possible:

- If $\alpha \geq \frac{d}{d-s^{\prime}(0)}$, then for all $a \geq \alpha, G(a)=a s(0)$ and $H(\alpha)=s(0)$. So,

$$
\operatorname{dim}_{H}\left(\left\{x: h_{f}(x) \leq s(0)\right\}\right)=d+s^{\prime}(0) .
$$

- If $1 \leq \alpha \leq a_{0}$ then

$$
\inf _{a \geq \alpha} G(a)=G\left(a_{0}\right)=\left(a_{0}\left(-q_{c} d+s\left(q_{c}\right)\right)+q_{c} d\right)=d q_{c} .
$$


and the corresponding value of $H$ is

$$
H(\alpha)=\frac{1}{\alpha} \inf _{a \geq \alpha} G(a)=\frac{d q_{c}}{\alpha}
$$

Thus, the spectrum of singularities is defined on the interval $\left[\frac{d q_{c}}{a_{0}}, d q_{c}\right]$ and for almost every function, and for all $H \in\left[\frac{d q_{c}}{a_{0}}, d q_{c}\right]$

$$
\operatorname{dim}_{H}\left(\left\{x: h_{f}(x) \leq H\right\}\right)=\frac{H}{q_{c}} .
$$

Furthermore, we have already seen that :

$$
s^{\prime}(q(a))=\frac{d a-d}{a}
$$

which is an increasing function. As $s^{\prime}$ is decreasing, the application $a \mapsto q(a)$ is itself decreasing. So, for $\alpha \leq a_{0}, q \geq q_{c}=\frac{1}{p_{c}}$ and :

$$
\operatorname{dim}_{H}\left(\left\{x: h_{f}(x) \leq H\right\}\right) \leq \inf _{p \geq p_{c}}(p H-\eta(p)+d) .
$$

- If $a_{0} \leq \alpha \leq \frac{d}{d-s^{\prime}(0)}$, which is equivalent to

$$
\inf _{a \geq \alpha} G(a)=G(\alpha)=\alpha \sup _{q}\left(-q d+s(q)+\frac{q d}{\alpha}\right) .
$$

We obtain :

$$
H(\alpha)=\sup _{q}\left(-q d+s(q)+\frac{q d}{\alpha}\right) .
$$

So for almost every function, for all $H \in\left[s(0), \frac{d q_{c}}{a_{0}}\right]$,:

$\operatorname{dim}_{H}\left(\left\{x: h_{f}(x) \leq H\right\}\right)=\inf _{p \geq p_{c}}(p H-\eta(p)+d) \leq \inf _{p \geq p_{c}}(p H-\eta(p)+d)$.

Furthermore, see [16], the spectrum of singularities of all functions of $V$ satisfies:

$$
d(H) \leq \inf _{p \geq p_{c}}(p H-\eta(p)+d) .
$$

This implies that the Hausdorff dimension of the set $\left\{x: h_{f}(x)<\right.$ $H\}$ is strictly less than $\frac{d}{\alpha}$. As proved in [14], there exists a measure $m_{\alpha}$ 
such that $m_{\alpha}\left(\left\{x: h_{f}(x) \leq H\right\}\right)>0$. But by definition of the Hausdorff dimension, $m_{\alpha}\left(\left\{x: h_{f}(x)<H\right\}\right)=0$. Then, $m_{\alpha}\left(\left\{x: h_{f}(x)=H\right)>0\right.$ and

$$
d(H) \geq \inf _{p \geq p_{c}}(p H-\eta(p)+d) .
$$

Proposition 8. For almost every function $f$ in $V$, the scaling function of $f$ satisfy:

$$
\eta_{f}(p)=\eta(p), \quad \forall 0<p<\infty
$$

Proof: As we have $V=\bigcap_{\varepsilon>0,0<p<\infty} B_{p, l o c}^{(\eta(p)-\varepsilon) / p, p}$, for any $f \in V$ the scaling function is greater than $\eta(p)$ for all $p$. Let $\tau>0$ and $p>0$ be fixed. We denote $\tau(p)=\frac{\eta(p)}{p}+\tau$. We first prove that the set

$$
M(p)=\left\{f \in V ; f \in B_{p}^{\tau(p), \infty}\right\}
$$

is a Haar null set. Let $g$ be the function defined by wavelet coefficients given by (24) and $P$ be the probe spanned by $g$. First, we check that $g$ does not belong to $B_{p}^{\tau(p), \infty}$. We write $\beta_{j}=d\left(1-\frac{j}{J}\right)$, where $J$ is defined as in (24). This term $\beta_{j}$ takes discrete values, spaced by $\frac{d}{j}$ and between 0 and $d$. As the function $s$ is a concave function and $0 \leq s^{\prime}(q) \leq d$ for all $q$, there exists, for $j$ large enough, a $\beta_{j}$ near from $s^{\prime}(q)$ such that the line given by $\tau(p)+\beta_{j}\left(\frac{1}{\tilde{p}}-\frac{1}{p}\right)$ is always above the graph of $s$. Thus

$$
\forall \tilde{p}>0 \quad \tau(p)+\beta_{j}\left(\frac{1}{\tilde{p}}-\frac{1}{p}\right)>s(1 / p) .
$$

But $a(j, k)=j \inf _{p}\left(\frac{\beta_{j}}{p}-s(1 / p)\right)$, and this infimum is attained for a $p_{0} \in(0, \infty)$. Therefore,

$$
a(j, k) \geq j\left(\frac{\beta_{j}}{p}-\tau(p)\right),
$$

and

$$
\|g\|_{B_{p}^{\tau(p), \infty}} \geq \sup _{j} j^{-a_{j} p} 2^{\tau j p}
$$

Thus $g \notin B_{p}^{\tau(p), \infty}$. 
Let $f$ be in $V$. Suppose that there exist $\alpha_{1}$ and $\alpha_{2}$ such that the functions $f_{1}=f+\alpha_{1} g$ and $f_{2}=f+\alpha_{2} g$ belong to $B_{p}^{\tau(p), \infty}$. Then $f_{1}-f_{2}$ also belongs to $B_{p}^{\tau(p), \infty}$. But

$$
f_{1}-f_{2}=\left(\alpha_{1}-\alpha_{2}\right) g
$$

As $g$ does not belong to $B_{p}^{\tau(p), \infty}$, we have then $\alpha_{1}=\alpha_{2}$. Thus $M(p)$ is Haar null.

Taking countable union over $\tau \rightarrow 0$ and $p>0$, we obtain that for almost every $f$ in $V, \eta_{f}(p) \leq \eta(p)$ for all $p>0$.

\section{References}

[1] P. Abry, Ondelettes et turbulences. Multirésolutions, algorithmes de décomposition, invariance d'échelle et signaux de pression, Nouveaux Essais. Paris: Diderot, 1997.

[2] A. Arnéodo, E. Bacry, and J.F. Mazy, The thermodynamics of fractals revisited with wavelets, Physica A. 213 (1995), 232-275.

[3] S. Banach, Über die Baire'sche Kategorie gewisser Funktionenmengen, Studia Math. 3 (1931), 174-179.

[4] J. Barral and B. B. Mandelbrot, Multifractal products of cylindrical pulses, Probab. Theory Relat. Fields 124 (2002), no. 3, 409-430.

[5] Y. Benyamini and J. Lindenstrauss, Geometric nonlinear functional analysis. Volume 1, Colloquium Publications. American Mathematical Society (AMS), 2000.

[6] G. Brown, G. Michon, and Peyrière J., On the multifractal analysis of measures, J. Stat. Phys. 66 (1992), 775-790.

[7] J.P.R. Christensen, On sets of Haar measure zero in Abelian Polish groups, Israel J. Math. 13 (1972), 255-260.

[8] A. Fraysse and S. Jaffard, How smooth is almost every function in a Sobolev space?, à paraître dans Rev. Math. Iber. Amer.

[9] _ How smooth is almost every function in a Sobolev space?, to appear in Rev. Mat. Iber. Amer. 
[10] U. Frisch and G. Parisi, On the singularity structure of fully developed turbulence, Turbulence and Predictability in Geophysical Fluid Dynamics and Climate Dynamics (R. Benzi M. Ghil and G. Parisi (North-Holland), eds.), pp. 84-88.

[11] J.-B. Hiriart-Urruty and C. Lemaréchal, Convex analysis and minimization algorithms. Part 1: Fundamentals., Springer- Verlag, 1993.

[12] B. Hunt, The prevalence of continuous nowhere differentiable function, Proceed. A.M.S 122 (1994), no. 3, 711-717.

[13] B. Hunt, T. Sauer, and J. Yorke, Prevalence: A translation invariant "almost every" on infinite dimensional spaces, Bull. A.M.S $\mathbf{2 7}$ (1992), no. 2, 217-238.

[14] S. Jaffard, Multifractal formalism for functions, SIAM J. Math. Anal 28 (1997), 944-970.

[15] _ Old friends revisited: The multifractal nature of some classical functions, J. Four. Anal. App 3 (1997), no. 1, 1-22.

[16] - On the Frisch-Parisi conjecture, J. Math. Pures Appl 79 (2000), 525-552.

[17] S. Jaffard and Y. Meyer, Wavelet methods for pointwise regularity and local oscillations of functions, Mem. Amer. Math. Soc. 123 (1996), no. 587.

[18] A. Kolmogoroff, The local structure of turbulence in incompressible viscous fluid for every large Reynold's numbers., C. R. (Dokl.) Acad. Sci. URSS, n. Ser. 30 (1941), 301-305.

[19] Y. Meyer, Ondelettes et opérateurs, Hermann, 1990.

[20] L. Olsen, A multifractal formalism, Adv. Math. 116 (1995), no. 1, $82-196$.

[21] E. Stein, Singular integrals and differentiability properties of functions, Princeton University Press, 1970. 